\title{
La formación docente en Argentina. Aproximación a un análisis político de la situación
}

\author{
Andrea Alliaud ${ }^{(1)}$
}

(1) Dra. en Educación por la Facultad de Filosofía y Letras, Universidad de Buenos Aires. Docente e investigadora del Instituto en Ciencias de la Educación. Facultad de Filosofía

\author{
y Letras, Universidad de Buenos \\ Aires. Profesora de posgrado en \\ la Universidad Torcuato Di Tella, \\ Argentina. Coordinadora del Área \\ de Formación Docente en la OEI \\ de Argentina.
}

\section{Introducción.}

En las últimas décadas la formación docente viene cobrando centralidad tanto en los ámbitos de producción de conocimientos especializados como en los de las políticas educativas. Distintos trabajos realizados en contextos diversos advierten sobre la necesidad de acompañar el cambio escolar con procesos de renovación del profesorado, aspecto considerado "crucial" en cualquier proceso de transformación escolar. ${ }^{(1)}$

En nuestro país se han desarrollado desde finales de la década de los 80 políticas tendientes al mejoramiento y fortalecimiento de la formación de docentes aunque, como en la mayoría de los países de la región, los resultados de las evaluaciones internacionales siguen manifestando un bajo rendimiento en relación a los contenidos mínimos referidos a las principales áreas del aprendizaje. Los problemas de cobertura, deserción y desgranamiento son asimismo deudas pendientes sobre todo en el nivel medio, ${ }^{(2)}$ obligatorio a partir de la nueva Ley de Educación Nacional. ${ }^{(3)}$

Si bien las políticas tendientes a la profesionalización de la docencia resultan prioritarias, no pueden ser consideradas con exclusividad a la hora de
(1) En: OeI, XIII Conferencia Iberoamericana de Educación. Plan de Cooperación. Renovación de Ideas y Formulación de Estrategias tendientes al fortalecimiento de Politicas de Formación Docente. Tarija, Bolivia, 4 y 5 de septiembre de 2003. (2) Si bien la tasa de escolarización del nivel medio ascendió al 71,46\%, sólo 1 de cada dos jóvenes arriba al último año de estudios en la edad correspondiente.

(3) Ley de Educación Nacional. Hacia una educación de calidad para una sociedad más justa. Ley 26206, enero de 2007. 
avanzar en la mejora educativa. En este sentido, una política educativa que no atienda en forma simultánea distintos aspectos corre el riesgo de evadir la complejidad estructural que caracteriza y explica la situación por la que atraviesan los sistemas educativos. Subiendo aún más la apuesta podrá decirse que las políticas educativas harán agua si no se integran con políticas económicas y sociales acordes (Alliaud, 2010).

En este marco me interesa presentar aquí un análisis del sistema formador de docentes en Argentina, tomando en consideración, en primer lugar, las características principales que la oferta formativa presenta para, en se marco, tratar de identificar las principales problemáticas que lo atraviesan. En segundo lugar, se hará referencia a las políticas educativas más recientes que, de un modo u otro, han tratado de superar los problemas existentes, sean éstos de índole estructural o coyuntural. Finalmente, se hará alusión a la experiencia internacional con la finalidad de identificar las respuestas o decisiones que algunos países han tomado para superar problemas similares a los de nuestro subsistema formador. En este punto reciben un tratamiento especial los temas de evaluación y carrera docente.

\section{Características de la oferta del subsistema formador de docentes.}

Históricamente en nuestro país la formación de docentes se desarrolló a través de dos circuitos paralelos: el "normalista" que nutría al sistema educativo formal y el "universitario" que formaba básicamente para las distintas profesiones $y$, en menor medida, también ofrecía titulaciones docentes. Las trayectorias educativas y sociales de los aspirantes a ambos circuitos formativos corrieron, hasta bien entrado el siglo XX, también por distintas vías:

En la concepción original los dos circuitos mencionados se expresan en que hay quienes tienen una preparación específica para el ejercicio docente: los profesores que trabajan en el circuito Normal - primaria; y que hay quienes no la tienen: los profesores enrolados en el circuito Bachillerato-Universidad, aunque sí poseen, en cambio, la 'preparación' apropiada desde el punto de vista social, el título y el prestigio universitario (Pinkasz, 1992:65). 
Hasta el presente coexisten en Argentina instituciones superiores universitarias y terciarias que se ocupan, bajo regímenes diferentes, de formar maestros y profesores. Aun así, los docentes con título universitario representan una minoría en el sistema educativo: en la Ciudad de Buenos Aires, por ejemplo, alcanzan el $25 \%$ en el nivel medio. En un estudio realizado por Mollis y otros (2006) se comparan los circuitos formativos terciarios y universitarios que ofrecen carreras docentes. Los resultados identifican la convivencia de distintos modelos de formación. Mientras las universidades priorizan la formación académica, en detrimento de la formación pedagógica (la cual aparece como un ciclo posterior a la licenciatura o como una diversificación de materias), en los institutos terciarios la formación docente constituye el eje vertebrador de la propuesta formativa. Existe, sin embargo, una diferencia, entre las propuestas terciarias que forman docentes para los niveles inicial y primario y las que forman para el nivel medio, basada en la especialización disciplinar. La excepción a los modelos "antagónicos" de formación docente la constituyen las universidades de más reciente creación, ${ }^{(4)}$ en las que se combina la investigación, como una manera de asegurar excelencia académica, y la centralidad en la formación pedagógica.

Asimismo, tal como lo demostraron distintas investigaciones (Davini, 2005; Aguerrondo y Vezub, 2008), los rasgos organizacionales del nivel superior no universitario dan cuenta del mantenimiento de una estructura o gramática escolar en cuanto a la forma en que se distribuyen los tiempos y espacios, los modos en que se plantea la relación con los conocimientos y los contenidos de la formación, los rituales, las maneras de plantear el vínculo pedagógico, el perfil de los formadores, las dinámicas de gobierno institucional, etc. No debe olvidarse que el nivel superior terciario depende, desde los años 90, de las respectivas jurisdicciones de gobierno educativo provincial, a diferencia de las universidades que si bien están bajo la órbita nacional son autónomas.

Dentro del circuito terciario, es decir, aquel que provee la mayor cantidad de docentes al sistema educativo formal, las instituciones formadoras (IFD) son en Argentina numerosas y divergentes en cuanto a oferta, localización,
(4) Nos referimos especialmente a las universidades creadas en el Conurbano Bonaerense a lo largo de las últimas dos décadas, además de las experiencias que puedan existir en las distintas jurisdicciones, tanto en el ámbito público como en el privado. 
(5) Según fuente: 320405 . Ministerio de Educación. Dirección Nacional de Información y Evaluación de la Calidad Educativa. Red Federal de Información Educativa. Años 2008-20Io.

(6) Ley 26075 , enero de 2006. cantidad de alumnado y tipo de formación que ofrecen. Dos problemas centrales pueden señalarse en torno a la oferta: uno de ellos, es la convivencia en una misma institución de carreras disímiles ya sean de formación docente o no; el otro, la desconexión que muchas veces acontece entre la formación de maestros y profesores. Así, a la falta de especialización de la mayoría de las instituciones, se suma la desarticulación entre carreras que presentan tramos formativos comunes.

La matrícula de los IFD presenta fluctuaciones debidas, la mayoría de las veces, a aspectos extrínsecos al subsistema formador. Las características del mercado laboral, las posibilidades y expectativas para conseguir empleo que poseen los jóvenes, son las variables principales que explican los movimientos matriculares que se presentan en el nivel terciario asociado, históricamente, con la posibilidad de obtener empleo seguro en poco tiempo. En el caso de la docencia podríamos añadir también la posibilidad de obtener un trabajo que aún conserva intactos los derechos y beneficios que caracterizaron al empleo público.

En la actualidad hay más de 300000 estudiantes $^{(5)}$ que aspiran a ser docentes; así y todo, se detectan problemas para cubrir cargos que el sistema educativo requiere en algunas jurisdicciones del país y/o en ciertas áreas disciplinares. Si bien progresivamente se van superando los bajos niveles de remuneración propios de la actividad (fundamentalmente a partir de la sanción de la Ley Nacional de Financiamiento Educativo) ${ }^{(6)}$ se añaden a la docencia, en el presente, crecientes dosis de desprestigio y desvalorización social, lo cual no estimula a los jóvenes a tomarla como una opción de estudio y empleo "valiosa". Junto con las condiciones materiales, el reconocimiento y valor simbólico de la profesión se presentan entre los desafíos a asumir por las políticas de formación.

Los rasgos mencionados explican no sólo los movimientos matriculares sino también la composición del alumnado que acude a los institutos de formación. Tal como lo señalan distintos estudios que abordan la temática, aquellos que eligen la docencia lo hacen como segunda opción, o como única opción que puede ofrecer mayores niveles de empleabilidad y calidad del empleo que el resto, como una posibilidad que brinda estabilidad laboral y movilidad ascendente (Davini, 2005:38-39). El cambio (real y per- 
cibido) en la composición de los alumnos del profesorado plantea dificultades a quienes se encargan de su formación. Tanto los directivos como los profesores, señalan que los ingresantes tienen problemas en las competencias básicas (Davini, 2005:35): capacidad de expresión, técnicas de estudio, etc. necesarias para brindar una base que posibilite una formación exigente y de calidad. Se han expuesto hasta aquí algunos problemas que podríamos llamar clásicos o típicos de la formación docente en nuestro país. En su mayor parte no son nuevos, por el contario, suelen remitir a los orígenes fundacionales de la profesión. Las políticas más recientes, llevadas a cabo en las últimas décadas, intentaron dar respuesta a ellos aunque, como veremos enseguida, en el caso de las desarrolladas en los años 90 generaron otros que, junto con los clásicos, fueron contemplados y asumidos por las políticas educativas implementadas a partir del gobierno kirchnerista (2003 hasta el presente).

\section{Políticas educativas recientes.}

Durante los años 90, dentro de un contexto de reforma educativa generalizada, las políticas de formación se orientaron fundamentalmente hacia el perfeccionamiento, reconversión y actualización de los docentes al considerar que era necesaria la apropiación por parte del cuerpo profesoral de los nuevos contenidos definidos para una "nueva" escuela. Si bien el Estado avanzó en la construcción de una "Red Federal para la Formación y Capacitación Docente Continua", los efectos formativos resultaron paradójicos sobre todo por el lugar en que quedaron posicionados los docentes. Asimismo, durante esta década fueron modificados los planes de formación inicial aunque, en este caso, la falta de compromiso hacia los acuerdos federales logrados, sumada a la transferencia abrupta de los servicios educativos del nivel superior a las provincias, produjo una fuerte fragmentación y desintegración del sistema formativo nacional. Cuando en nuestro país se hace efectiva la transferencia de la educación superior a las provincias, si bien se definen acuerdos legales, normativos y pedagógicos, el carácter no vinculante de los mismos acentúa la escasa integración existente en el sistema formador de docentes, provocando la coexistencia de sub-sistemas con características muy divergentes entre sí, llegando incluso 
(7) Se han contabilizado en nuestro país más de 6000 carreras docentes, muchas de las cuales dan títulos semejantes pero no idénticos

(Aguerrondo, Vezub y Clucellas, 2008).

(8) Un estudio sobre la evolución de los salarios docentes, citado por Birgin (1999), indica que en los 90 el sueldo de un maestro de grado correspondía al 2I,4\% de lo que era en 1935 . Como efecto de la política de "congelamiento", en 1996 Argentina estaba entre los tres países de América latina con mayor deterioro de lo salarios docentes

(9) (Birgin,I999:80-8I). En un diario de la época (Página I2, Io/o7/I998), se lee que

el ministro de Economía afirmaba: "los maestros ganan poco porque trabajan poco". a no ser reconocidos los títulos otorgados entre una jurisdicción y otra. ${ }^{(7)}$ Según Davini (2005) la transferencia no se acompañó de una configuración organizativa, sino que reguló básicamente a nivel del curriculum y la institución.

Un rasgo característico de la región fue la implementación de este tipo de políticas definidas como "modernizadoras", dentro de un panorama general de reducción del gasto público y achicamiento del Estado. En lo que refiere específicamente a la docencia, se da así la paradoja que el discurso de la "profesionalización" habita en un contexto de empeoramiento de las condiciones laborales. Si bien los docentes nunca han tenido los mejores salarios, los datos indican que durante estos años se ha llegado a pisos históricos de deterioro. ${ }^{(8)}$

Otro rasgo característico de las políticas educativas "neoliberales" fue el papel protagónico que las reformas otorgaron a agentes "externos" a las instituciones educativas. La definición de contenidos curriculares estuvo a cargo de especialistas, a partir de los avances producidos en los distintos campos del conocimiento científico. De este modo, "la división del trabajo intelectual en el interior del campo quedaba bien delimitada y determina qué saber es legítimo, cabiéndole a los docentes la reproducción del conocimiento generado por unos y organizado por otros" (Davini, 1998:84). Esta tendencia también estuvo presente en la definición de los nuevos planes de formación docente y en las propuestas de capacitación. Mientras los contenidos para la formación inicial avanzaban en prescripciones y especificidades, dejando escaso margen para la "recontextualización" curricular en las instituciones, las propuestas de formación continua proliferaron bajo el formato de cursos acreditados, mediante los cuales los docentes tenían que "perfeccionarse", "actualizarse", "reciclarse", suponiendo que todo lo que habían hecho y aprendido de su quehacer resultaba desechable o perjudicial en los nuevos escenarios escolares. De este modo, el docente fue colocado y se colocó en el lugar del "no saber"(9) y fue ello lo que justificó la necesidad de capacitación masiva. "El énfasis en el acceso al conocimiento de los especialistas, se produce en detrimento del saber peculiar de los docentes y a favor del saber producido por otros" (Davini, 1998:85). 
Debido a la situación existente y a partir de acuerdos federales alcanzados por el nuevo gobierno nacional, en el año 2006 el Ministerio de Educación crea el Instituto Nacional de Formación Docente (INFD), un organismo nacional desconcentrado, cuya función primaria es la de planificar, desarrollar e impulsar políticas de formación docente inicial y continua. Las funciones de este Instituto Nacional se explicitan en el artículo 76 de la Ley de Educación Nacional en el que se añaden, a la planificación y ejecución de políticas, las responsabilidades de: fortalecer las relaciones entre el sistema formador y el sistema educativo; aplicar las regulaciones que demande la organización del sistema; promover lineamientos curriculares básicos para la formación docente inicial y continua; impulsar y desarrollar acciones de investigación y desarrollo curricular; coordinar acciones de seguimiento y evaluación de las políticas e impulsar acciones de cooperación técnica interinstitucionales e internacionales. Además de garantizar la conformación de un "sistema formador" con una institucionalidad propia, la ley extiende la duración de las carreras docentes a cuatro años ${ }^{(10)}$ e intenta garantizar las Residencias prolongadas.

La convivencia de la calidad educativa con la justicia social, como principios básicos de la norma, se plasma en las finalidades otorgadas a la formación docente: "La formación docente tiene la finalidad de preparar profesionales capaces de enseñar, generar y transmitir los conocimientos y valores necesarios para la formación integral de las personas, el desarrollo nacional y la construcción de una sociedad más justa" (Art. 71).

Finalmente, la nueva ley nacional avanza sobre otros aspectos pendientes en nuestro país referidos al trabajo docente. Entre ellos se pueden destacar: la enunciación conjunta del mantenimiento de la estabilidad laboral y el acceso a los cargos por concursos de antecedentes y oposición, la definición de la carrera docente que admitirá al menos dos opciones (desempeño en el aula y desempeño de la función directiva y de supervisión) y la formación continua como una de las dimensiones básicas para el ascenso. Respecto a las condiciones laborales y también a las prioridades otorgadas a la formación de los docentes la Ley de Financiamiento Educativo establece las pautas que aseguran un incremento progresivo en la inversión educativa.
(10) Hasta el momento los planes de formación docente para los niveles inicial y primario (a diferencia de los de media) tenían una duración estimada de tres años en la mayoría de las provincias del país. 
El marco legal vigente plantea metas y aspiraciones, desafíos importantes a la vez que crea garantías, legitima realidades y genera condiciones de posibilidad. Las líneas de acción que se están llevando a cabo actualmente en materia de formación y desarrollo profesional docente, cuentan con un órgano rector de las políticas de Estado que avanza sostenidamente en la direccionalidad establecida, a través de acuerdos "vinculantes" que se van alcanzando con las distintas provincias y con representantes de universidades, gremios y otros actores relevantes de la gestión educativa.

Los problemas que quedaron por delante, sobre los que se está avanzando progresivamente, se refieren básicamente al desarrollo de una institucionalidad específica que contemple la formación inicial y continua de los docentes en función de la mejora de las escuelas. Temas vinculados con la planificación de la oferta formativa y una mayor articulación de estas instancias con la carrera profesional; la producción de saberes sobre la enseñanza y una pedagogía específica para la formación; la articulación con las universidades en tanto instituciones que también forman docentes y producen conocimientos sistemáticos; los procesos de seguimiento y evaluación permanentes, la definición de marcos normativos específicos que regulen la educación superior merecen, entre otros, una atención especial.

Desde los comienzos del INFD se planteó la necesidad de definir lineamientos políticos, institucionales y pedagógicos que, acordados y comprometidos federalmente, avancen hacia el fortalecimiento de un sistema formador integrado en base a una identidad propia del nivel superior terciario que combine, desde nuestra perspectiva, excelencia académica con formación para el trabajo (escolar). En este sentido, la formación docente tendría que incorporar en su desarrollo las características y los escenarios de realización del puesto de trabajo, siendo la especificidad del mismo los procesos de enseñanza. Entre los acuerdos federales recientes, figura un Documento elaborado por el INFD en el que se menciona "la preocupación por el desarrollo de una institucionalidad específica del sistema formador, que le permita superar la atomización y recibir un impulso configurador a través de la planificación" (Terigi, 2007:5). La planificación del sistema de formación docente, tanto inicial como permanente, requiere considerar las necesidades del sistema educativo para el que 
se forma. Esta perspectiva incluye tanto la provisión de docentes como el desarrollo profesional de los que están en ejercicio, en función de las necesidades actuales y de las que surjan a partir de los desafíos que deben afrontar los distintos niveles educativos (básicamente referidos a la extensión de la escolaridad obligatoria estipulados por ley, pero también contemplando las transformaciones que pretenden impulsarse en las escuelas). En nuestro país, la planificación de la oferta debería considerar las necesidades de cada provincia y sus reales posibilidades de abrir nuevas carreras (Davini, 2005:64-65). Considerar las particularidades provinciales no implica, sin embargo, que la planificación no sea a escala del sistema y no necesariamente a escala provincial (Terigi, 2007).

Además de la oferta, existe una arista pedagógica en el problema de la vinculación entre el sistema de formación y el sistema educativo referida a la producción de saberes específicos sobre la enseñanza, sobre el trabajo docente y la formación. La planificación del sistema formativo y su orientación hacia el sistema educativo, tiene como contrapunto la evaluación, en tanto mecanismo que permite asegurar, mejorar, corregir, la direccionalidad de la formación que se imparte.

A nivel del sistema formador resultaba igualmente importante establecer cierta base normativa común que regulara tanto la formación inicial de los docentes como el desarrollo profesional y permanente. En el primer caso se ha avanzado, desde el ámbito nacional, hacia la definición de "Lineamientos Curriculares Comunes" que recuperan las modificaciones estipuladas por ley en cuanto a la extensión a un mínimo de cuatro años de todas las carreras ${ }^{(11)}$ y avanzan en la definición de una propuesta formativa orientada fundamentalmente hacia la práctica escolar docente sin descuidar los fundamentos generales ni los conocimientos específicos sobre las disciplinas y las problemáticas propias del nivel de enseñanza de desempeño. En el segundo caso queda pendiente la definición de un marco orientador de la formación continua y el perfeccionamiento de los profesores que, articulado con la formación inicial, resulte superador de la dispersión y fragmentación de la oferta actualmente existente y de los problemas de ingreso, retención y deserción de la profesión que también afectan al sistema formador. Las definiciones
(11) En cuanto a la formación inicial, en varios países ésta equivale al grado universitario, lo que posibilita el acceso a estudios de maestría o doctorado. 
(12) Por tratarse de un tema específico le dedicaremos al mismo un apartado especial. en este sentido, deberían incluir especialmente el acompañamiento durante los primeros desempeños, la formación en servicio y tendrían que vincularse con el establecimiento de la carrera docente. ${ }^{(12)}$

Otro de los elementos que merecen atención a nivel del sistema formador se refiere al logro de una articulación real y efectiva del nivel superior permitiría rescatar la fortaleza de los modelos vigentes y desafiar, a unas y otras instituciones, sobre el desarrollo de sus deudas pendientes. Para ello resulta imprescindible pensar y regular la formación docente como un sistema integrado, considerando la totalidad de las instituciones que lo integran. Tanto los espacios de definición de políticas, como las mismas universidades tienen una responsabilidad ineludible en este aspecto.

Tomando en cuenta las fortalezas y debilidades de los modelos vigentes, sería posible, en principio, avanzar en la articulación, enunciada en la Ley de Educación Superior (24521), considerando "los diferentes tipos de instituciones que la integran" (Art. 4, inc. F). Más allá de este enunciado general, queda pendiente una norma vinculante y general que regule las condiciones que harían posible una verdadera articulación en el sistema formativo. Contamos por el momento con actividades emprendidas por instituciones y actores particulares que podrían tomarse como insumo. Entre ellas se pueden mencionar: el reconocimiento de materias cursadas por los alumnos en uno u otro circuito, las equivalencias de los concursos docentes, la realización de posgrados, el desarrollo de proyectos conjuntos de investigación, extensión, entre otros.

\section{La tendencia internacional}

Las experiencias internacionales en materia de formación docente, brindan algunos aportes para considerar en función de las problemáticas que se vienen analizando. Una vez más, no se trata de tomar lo ajeno para replicarlo mecánicamente dejando de lado tradiciones, historias y procesos locales. La intención de aludir a estas experiencias es la de "abrir un abanico" de posibilidades que permita enriquecer los procesos de toma de decisiones y las prácticas en el ámbito nacional. 
Una breve panorámica de la experiencia internacional muestra que en la mayoría de los países la formación de los docentes viene siendo asumida por las universidades o por instituciones que adoptan los parámetros de la actividad universitaria. Tal es el caso, por ejemplo, de los IUFM franceses que reemplazaron a las escuelas normales. Así y todo, no existe acuerdo generalizado de pasar la formación docente a las universidades. El pedagogo británico Ivor Goodson sostiene que la preocupación por la práctica escolar, propia de la formación de profesores, se vio desdibujada con el traspaso a la universidad frente a la aspiración del logro de status profesional siguiendo los parámetros del trabajo académico más convencional. Una vez más esta disyuntiva pone de manifiesto la "disociación" existente entre la formación para el trabajo (escolar) y la excelencia académica.

La experiencia internacional demuestra que el pasaje de la formación a la universidad no es en sí mismo suficiente para mejorar la docencia, a menos que se contemplen otras variables. El caso de Finlandia, actualmente unos de los países más avanzados en cuanto a resultados educativos y por ello frecuentemente citado, demuestra que además traspasar completamente la formación de profesores a la universidad, la transformación iniciada en los años setenta incluyó: la unificación de la formación docente, planes de estudio que incrementaron el peso de las ciencias de la educación y también de las ciencias sociales y políticas (lo que aquí llamamos generales), la selección rigurosa de los estudiantes y el fortalecimiento de la formación docente continua a través de carreras e posgrado y tesis de maestría. El mejoramiento salarial fue otra de las variables que se contempló entre las políticas educativas implementadas. En segundo lugar se advierte en la mayoría de los países del mundo un aumento en la duración de las carreras docentes equiparándose con la duración de cualquier carrera de grado superior universitario, habilitando a los docentes para cursar estudios de posgrado, maestrías o doctorados. Actualmente la duración de las carreras docentes va desde los dos o tres años (o el equivalente a una educación postsecundaria) hasta los 8 años como en el caso de Alemania. Existen excepciones donde se titula y habilita para trabajar con una formación de nivel secundario. Se ha establecido una clasificación internacional, la Internacional Standard Classification of Education, que identifica distintos modelos o tipos de formación según el nivel educativo requerido para 
enseñar. Este requisito junto con la proporción de docentes titulados, constituye uno de los indicadores más frecuentes de la calidad docente.

En tercer lugar, la tendencia internacional direcciona hacia la unificación de carreras que forman docentes para los distintos niveles de enseñanza; superando así la vigencia de modelos que asocian los años de formación con el nivel de escolaridad del desempeño. La nueva Ley Nacional de Educación de nuestro país avanzó en este sentido al llevar a cuatro años la formación de docentes para los niveles primario e inicial, equiparándola así con la formación de profesores de nivel secundario, no así con las carreras universitarias. En cuarto lugar, muchos países han aumentado los niveles de autonomía institucional en la gestión curricular y se han impulsado cambios en los formatos "tradicionales" de formación, impulsados frecuentemente por la escasez de docentes. En el continente europeo, se encuentran desde programas a distancia, apoyados en el uso de las nuevas tecnologías, programas de "certificación alternativa" que reconocen antecedentes formativos en otras áreas hasta la "certificación en el empleo", basados en la evidencia. El caso excepcional, dentro de los países europeos, es el de Alemania que se destaca por la importante centralidad que el Estado mantiene en la definición de los contenidos y la duración de los programas de formación. En muchos de los otros países, son las propias instituciones (institutos o universidades) las que ofertan carreras docentes siendo sometidas a fuertes procesos de acreditación y evaluaciones de seguimiento por parte de los Estados. Para Iberoamérica, el caso más parecido a estos modelos es el chileno.

En nuestro país los acuerdos federales existentes refieren al nivel de autonomía de las instituciones terciarias respecto del Estado. "Se debe tener presente que el Estado es el garante de la autonomía y es también el garante del cumplimiento de los propósitos y funciones públicamente asignadas al sistema formador y a las escuelas" (Resolución CFE No 30/07 Anexo I). En este sentido, se señala la necesidad que las instituciones superiores se den sus propias normas a partir de una normativa común que garantice el carácter doblemente público de la formación docente. El ámbito de lo "común" para la formación docente local incluye formas de gobierno electivas y colegiadas para las instituciones así como procesos de participación estudiantil. 
Para finalizar, el rastreo de la experiencia internacional es necesario referir a dos cuestiones que en nuestro medio resultan polémicas tales como son la evaluación y la carrera docente. Dedicaremos a ellas un apartado especial.

\section{Evaluación y carrera docente}

En lo que refiere particularmente a la carrera docente, se trata de superar la actual estructura piramidal y de una sola vía por la que se "avanza" en el circuito laboral docente, y estipular distintas opciones de carrera profesional (lo expresado en la Ley de Educación Nacional constituye un punto de partida), relativizando la preponderancia fundamental de la antigüedad para alcanzar ascenso e incremento salarial. ${ }^{(13)}$ La movilidad en la carrera docente, podría depender de la incorporación de nuevas tareas que permitan ensanchar el ámbito de actuación dentro de un mismo puesto; o el cambio de categoría y ascenso que conlleva el ejercicio de nuevas funciones y responsabilidades.

Actualmente, el desarrollo profesional de los profesores no se correlaciona con las posibilidades mencionadas. Por el contrario, las oportunidades de crecimiento y ampliación de responsabilidades son muy limitadas debido a la verticalidad de la carrera, en la cual la única posibilidad de promoción es mediante el alejamiento del aula a través del acceso a cargos de conducción. De este modo, los ascensos e incrementos salariales se consiguen cambiando de función y/o de nivel de enseñanza (como ocurre por ejemplo con los profesores de secundaria que pasan a trabajar al nivel superior). ${ }^{(14)}$ Algunas de las vías de superación que han encontrado los distintos países para salvar las cuestiones planteadas, son las siguientes:

- Subsidio para las residencias docentes, lo cual además de mejorar las condiciones formativas establece un eslabón con la carrera laboral.

- Subsidio para el ejercicio en escuelas desfavorecidas.

- Subsidios para el ejercicio de otras funciones tales como tutorías, mentorías a docentes noveles por parte de maestros y profesores en ejercicio. - Subsidios para el ejercicio de tareas de coordinación de áreas, ciclos, etc.
(13) Tanto en EE. UU. como en Europa, los incrementos salariales de los docentes están aún muy asociados con la antigüedad (Vaillant, 2004).

(14) Ver Aguerrondo y Vezub, 2008. 
- Incentivos salariales ligados con el desempeño. Los mismos se aplican a nivel nacional, provincial o municipal, así como dotando a las instituciones escolares de mayores niveles de autonomía tanto para la evaluación como para la toma de decisiones acerca de lo que hacen.

Los subsidios que reciben los docentes por la ampliación de sus tareas, el incremento de sus responsabilidades y/o la especialidad de sus funciones, se vinculan en estos casos con trayectos de capacitación específicos diseñados a partir de las necesidades y los cambios que los puestos laborales requieren. En Noruega, por ejemplo, los aumentos salariales se vinculan con los resultados y el desempeño individual de los docentes pero existen, además, programas de iniciación a la docencia que tienen por finalidad evitar la deserción de la carrera. La figura del "mentor", elegido entre los docentes de una escuela, se destina al acompañamiento de los nuevos docentes. Estos perfiles, junto con el personal directivo de la institución, reciben capacitación específica, así como los docentes noveles comparten ámbitos de formación con otros colegas en redes coordinadas por las instituciones formadoras (GTD/ PREAL No 15, 2006).

Los aspectos relativos al establecimiento de la carrera docente llevan a reparar en un aspecto relevante y polémico de la gestión del sistema educativo: la evaluación docente. La preponderancia que la misma ha adquirido en el último tiempo se vincula con la necesidad de realizar seguimientos y monitoreos periódicos con la finalidad de "mejorar" la práctica de los docentes y a partir de ella la calidad de la educación que se imparte. Si bien desde esta perspectiva se asocia automáticamente la evaluación al desempeño docente, medido en términos de resultados de aprendizaje de los alumnos, cabe señalar que ésta es sólo una de las formas posibles de concebir los procesos de evaluación, de ninguna manera es la única.

La tendencia mundial indica que los países utilizan distintos y diversos mecanismos de evaluación y certificación de las prácticas docentes, según focalicen en:

- la formación inicial y continua;

- el ingreso a la carrera;

- el ingreso a la profesión. 
Antes de la obtención del título docente se puede exigir desde la aprobación de un examen hasta haber pasado un período de prueba en instituciones educativas. En Francia, por ejemplo, donde se exige una licenciatura para ingresar a la carrera docente, los candidatos son evaluados luego de dos años donde se preparan pedagógicamente; sólo la aprobación posibilita la obtención del diploma docente. En EE. UU. se tomaban exámenes escritos para otorgar titulaciones docentes y en algunos casos se implementaban exámenes durante los primeros años de desempeño profesional. En Inglaterra y los países vecinos se han establecido competencias necesarias para la enseñanza, a partir de las cuales se organiza la formación inicial y se establecen los requisitos para evaluar los inicios y las sucesivas etapas de la carrera docente. Se trata en este caso de sistemas de certificación periódicos definidos a escala nacional.

Existen, además, distintos niveles de "evaluación del desempeño" docente que han sido tomados por los distintos países ${ }^{(15)}$ en particular o de manera combinada. En estos casos la evaluación puede realizarse considerando:

- el perfil profesional de los docentes, a través de su formación inicial y permanente;

- Ios resultados de aprendizaje obtenidos por los alumnos;

- los comportamientos de aula del docente (estrategias, vínculos con los alumnos, el saber, etc.);

- los vínculos que establece el docente con otros colegas.

En Australia los docentes se evalúan en distintos momentos de su carrera y a través de diversos mecanismos durante un período. Establecido el diagnóstico inicial y en función de estándares definidos, se diseñan planes de desarrollo profesional. Una vez finalizada la etapa de formación de grado o inicial, algunos países como Canadá, otorgan certificaciones provisorias para el ejercicio profesional y luego de una etapa de seguimiento y evaluación del desempeño (dos años) se obtiene el título permanente. A lo largo de su carrera y con una frecuencia anual, cada docente debe preparar un plan de desarrollo profesional que es evaluado por el director de su escuela. EE. UU. es el país en el que se relaciona la acreditación de los
(15) Ver Vaillant (2004). 
docentes con los avances logrados por los alumnos, tomando en consideración no tanto los resultados obtenidos como la mejora alcanzada a lo largo del ciclo lectivo. También en muchos estados se observan clases y/o se exige la preparación de materiales por parte de los docentes.

En América Latina es poco lo que se ha avanzado al respeto, aunque la preocupación está instalada y a partir de allí en los últimos años se promovieron en la región modelos llevados a cabo en otros contextos. La excepción la constituye Chile, que descentralizó su sistema educativo y donde los establecimientos reciben incentivos como "subvenciones de excelencia", a partir de comparar su rendimiento con otros de características similares. En el año 2004 se aprobó en el país andino una ley sobre evaluación docente, en el marco de la llamada "buena enseñanza", definida operacionalmente e integrando el análisis al contexto de referencia.

Para el caso argentino, es importante considerar que la docencia conserva intactos, como se dijo, los rasgos principales del "empleo público", tales como la estabilidad laboral y el aumento salarial por antigüedad. Existen Estatutos docentes que regulan fuertemente la actividad. Pero el sistema vigente fomenta el credencialismo, siendo la acumulación de certificaciones la única forma de obtener mayor puntaje para titularizar y poder ascender, además de la antigüedad en el cargo. Hasta la nueva Ley de Educación Nacional, la única vía de ascenso para la carrera docente representaba la salida del aula.

Las aproximaciones más críticas se basan en los riesgos que la evaluación estandarizada conlleva. Entre ellos: el establecimiento de la correlación directa que suele establecerse entre el desempeño de los docentes (su evaluación) y los resultados de aprendizajes obtenidos por los alumnos, la dificultad de captar o medir competencias o capacidades, la complejidad de implementar un sistema que sea coherente con lo que aspira medir, los peligro de descontextualizar y aislar la práctica y el trabajo docente considerando exclusivamente el nivel del aula. Como síntesis de lo que se viene haciendo en materia de evaluación docente, podríamos decir que la experiencia internacional indica que es recomendable considerar en las evaluaciones más de un aspecto y diseñar pruebas piloto, antes de tomar medidas a escalas mayores. Asimismo, se recomienda priorizar procesos de autoevaluación institucional y participación del cuerpo docente tanto como de otros actores relevantes. 
La mayoría de los países realizan algún tipo de seguimiento en algún momento de la carrera docente, ya sea en el ingreso a la formación profesional, durante el proceso formativo, al finalizar el mismo y/o durante el desempeño. En algún momento se aplica algún mecanismo que permite distinguir tanto a aquellos que lo hacen bien como a los que no. La evaluación posibilita, por su parte, explicitar lo que está implícito con la voluntad colectiva de comprender lo que ocurre para generar decisiones que mejoren los sistemas educativos y las escuelas. La evaluación, bien entendida, no tiene por función principal establecer escalas jerárquicas ni sistemas de incentivos; más bien, se destina a conocer, verificar, monitorear y así poder decidir. Claro que implica resultados, valoración... Distinción sin humillación, pero tampoco dejando de lado la responsabilidad de todo sistema educativo de educar/formar/enseñar a todos. Al finalizar esta presentación estamos en condiciones de afirmar que no hay formas "puras" ni recetas mágicas que en sí mismas instalen la mejora. Para que los cambios incidan en el sistema formador, como en cualquier otro ámbito, se requiere de políticas integrales que no obvien las tradiciones, la historia, los modos de actuación y el saber de los actores. Mirar hacia afuera nos permite apreciar que sistemas educativos muy distintos en cuanto a su ideología, formas de organización y administración presentan altos niveles de rendimiento educativo.

Más allá de acordar o cuestionar los indicadores habituales de éxito escolar y de considerar las distintas variables puestas en juego en la producción de ciertos resultados, es preciso reconocer que sistemas educativos muy diferentes tienen un rendimiento comparable. Los tres países que encabezan la encuesta PISA (Finlandia, Japón y Corea del Sur) son un ejemplo de ello. Mientras el primero funciona con sistemas de clasificación cualitativos, programas personalizados en aulas heterogéneas (hasta los dieciséis años), con talleres, etc.; los segundos son muy selectivos, con un ritmo escolar extremadamente exigente, etc. Aún con profundas diferencias, lo común entre estos países está dado por la coherencia y la autenticidad en lo que hacen. En los sistemas más abiertos y democráticos, los docentes han desarrollado procesos de mejora, en el marco de una cultura de colaboración donde la comprensión y el compromiso por la calidad de lo que ocurre y se produce, incluye a todos. 
Alliaud, A. (2010). "La formación docente. Estado de situación y desafíos a futuro". Voces del Fénix. Revista del Plan Fénix, Año 1, $N^{\circ} 3$, septiembre. Disponible en: www.vocesenelfenix.com

Aguerrondo, M.I. y Vezub, L. (2008). Los profesorados de formación docente. Características de los formadores y de las instituciones. Una mirada hacia el interior de las instituciones terciarias de Formación Docente en la Argentina. Cambios y continuidades en su configuración. Buenos Aires: Universidad de San Andrés/Fundación Lúminis.

Aguerrondo, I.; Vezub, L. y Clucellas, M. (2008). Caracterización de las instituciones de educación superior con oferta de formación docente. Parte I: Las instituciones terciarias de formación docente, datos del RA 2004". UNESCO/IIPE Buenos Aires - INFoD/ME.

Birgin, A. (1999). El trabajo de enseñar. Entre la vocación y el mercado: las nuevas reglas del juego. Buenos Aires: Troquel.

Bolívar Botía, A. (2004). "La educación secundaria obligatoria en España: En la búsqueda de una inestable identidad". Revista Electrónica Iberoamericana de Calidad, Eficacia y Cambio en Educación, Vol. 2, № 1.

Davini, M.C. y Birgin, A. (1998). "Políticas de formación docente en el escenario de los 90 . Continuidades y transformaciones". En AA VV. Políticas y Sistemas de Formación. Formación de Formadores. Serie Los Documentos № 8. Buenos Aires: Facultad de Filosofía y Letras UBA/Novedades Educativas.
Davini, M.C. (2005). Estudio acerca de la calidad y cantidad de oferta de la formación docente, investigación y capacitación en la Argentina. Ministerio de Educación. Dirección Nacional de Gestión Curricular y Formación Docente. Agosto.

GTD -PREAL/ORT. "Programa de Promoción de la Reforma Educativa en América Latina y el Caribe2. Período 2006-2008.

Ministerio de Educación de la Nación (2007). Plan Nacional de Formación Docente. Instituto Nacional de Formación Docente (INFD). Marzo.

Mollis, M. et al. (2006). La formación universitaria para el sistema educativo y el sector productivo. Casos comparados. Buenos Aires: Planeta.

Pinkasz, D. (1992). "Orígenes del profesorado secundario en la Argentina". En Braslavsky, C.; Birgin, A. (comps.). Formación de Profesores - Impacto, pasado y presente. Buenos Aires: Miño y Dávila Editores.

Vaillant, D. (2004). “Construcción de la Profesión Docente en América Latina. Tendencias, temas y debates". PREAL n ${ }^{\circ} 31$. Diciembre.

Terigi, F. (2007). Hacia un acuerdo sobre la institucionalidad del sistema de formación docente en Argentina. Ministerio de Educación de la Nación. Instituto Nacional de Formación Docente (INFOD). Noviembre. 